\title{
Leitlinien in der Praxis: der herzchirurgische Patient in der intensivmedizinischen Versorgung
}

David Sander, Fabian Dusse

\section{Die S3-Leitlinie zur intensivmedizinischen Versorgung herzchirurgischer Patienten - Hämodynamisches Monitoring und Herz-Kreislauf [1] vereint aktuelles Wissen zu Empfehlungen für das hämodynamische Monitoring und die differenzierte Kreis- lauftherapie bei herzchirurgischen Intensivpatienten. Zudem werden anzustrebende Zielparameter der Kreislauftherapie, Behandlungsstrategien bei Links- und Rechts- herzinsuffizienz sowie klare Handlungsempfehlungen nach diversen Eingriffen in diesem Patientenkollektiv aufgezeigt.}

\begin{tabular}{|c|c|}
\hline \multicolumn{2}{|c|}{ ABKÜRZUNGEN } \\
\hline ACB & aortokoronarer Bypass \\
\hline BGA & Blutgasanalyse \\
\hline ECLS & Extracorporeal Life Support \\
\hline ESC & European Society of Cardiology \\
\hline etCO $\mathrm{C}_{2}$ & endtidaler $\mathrm{CO}_{2}$-Partialdruck \\
\hline $\mathrm{Hb}$ & Hämoglobin \\
\hline LAD & linke Koronararterie \\
\hline LCOS & Low cardiac Output Syndrome \\
\hline LIMA & Arteria thoracica interna sinistra \\
\hline LV & linksventrikulär \\
\hline NIBP & nichtinvasive Blutdruckmessung \\
\hline $\mathrm{paCO}_{2}$ & $\mathrm{CO}_{2}$-Partialdruck \\
\hline PAK & Pulmonalarterienkatheter \\
\hline $\mathrm{PaO}_{2}$ & Sauerstoffpartialdruck \\
\hline pAVK & $\begin{array}{l}\text { periphere arterielle Verschluss- } \\
\text { erkrankung }\end{array}$ \\
\hline PLR & Passive Leg Raise Test \\
\hline PVR & pulmonalvaskulärer Widerstand \\
\hline RV & rechtsventrikulär \\
\hline $\mathrm{ScvO}_{2}$ & zentralvenöse Sauerstoffsättigung \\
\hline $\mathrm{SpO}_{2}$ & $\begin{array}{l}\text { Sauerstoffsättigung (pulsoxymetrisch } \\
\text { gemessen) }\end{array}$ \\
\hline $\mathrm{SvO}_{2}$ & gemischt-venöse Sauerstoffsättigung \\
\hline v-a-ECMO & $\begin{array}{l}\text { venoarterielle extrakorporale Membran- } \\
\text { oxygenierung }\end{array}$ \\
\hline ZVDk & zentraler Venendruck \\
\hline ZVK & zentraler Venenkatheter \\
\hline
\end{tabular}

Ziel des vorliegenden Artikels ist die praxisrelevante Auseinandersetzung mit der Leitlinie [1] und Darstellung der relevantesten Empfehlungen zur direkten klinischen Umsetzung in der eigenen täglichen intensivmedizinischen Tätigkeit. Um alle Empfehlungen und deren Evidenzlagen zu erhalten, bleibt das direkte Studium der Leitlinie jedoch unabdingbar.

\section{Basismonitoring}

Das kardiopulmonale Basismonitorig inklusive des metabolischen Monitorings umfasst die grundlegenden Methoden zur Überwachung der Homöostase eines Intensivpatienten. Es beinhaltet

- EKG,

- die nichtinvasive Blutdrucküberwachung (NIBP),

- die invasive Blutdruckmessung,

- $\mathrm{SpO}_{2}$,

- etCO

- Temperatur und

- Urinausscheidung.

Das Basismonitoring stellt ein fest verankertes Konzept in der Intensivtherapie dar und sollte auch bei jedem postoperativen herzchirurgischen Intensivpatienten angewendet werden $[2,3]$.

EKG: Die frühzeitige Ischämiediagnostik mittels EKG-Monitoring ist bei herzchirurgischen Intensivpatienten von besonderer Relevanz. Hierzu dient neben einem 12-KanalEKG bei Aufnahme die kontinuierliche EKG- und ST-Strecken-Überwachung mittels mindestens 5-poliger EKG-Ableitung (II und $V_{5}$ ) [4]. Durch die Registrierung zusätzlicher Brustwandableitungen werden die Sensitivität der Diagnostik erhöht und das frühzeitige Erkennen einer kardialen Ischämie ermöglicht. Auch ist ein kontinuierliches EKGMonitoring für das Erkennen von nicht selten auftretenden postoperativen Rhythmusstörungen bei herzchirurgischen Patienten essenziell, da diese mit hämodynamischen Konsequenzen verbunden sein können. Somit sollte in der täglichen Praxis eine entsprechende EKG-Überwachung etab- 
liert, kontinuierlich durchgeführt und besonders aufmerksam evaluiert und dokumentiert werden, um eine ischämische Komplikation frühzeitig zu erkennen.

Blutdruckmessung: Ebenfalls zum Basismonitoring zählt die kontinuierliche invasive Blutdruckmessung. Mit ihrer Hilfe können der Blutdruck und hämodynamische Veränderungen zuverlässig registriert werden $[5,6]$. Die regelmäßige Abnahme einer arteriellen Blutgasanalyse ermöglicht neben der Überwachung der Oxygenierung $\left(\mathrm{paO}_{2}\right)$ und Ventilation $\left(\mathrm{paCO}_{2}\right)$ die Evaluation des SäureBasen-Haushaltes, der Elektrolyte, des Laktatwertes und des Glukosestoffwechsels. Diese Möglichkeiten der Überwachung von arteriellem Blutdruck und Blutgasanalysen machen die invasive Blutdruckmessung besonders bei komplikativen Verläufen auch längerfristig unentbehrlich. Bei komplikationslosem Verlauf kann die invasive Blutdruckmessung am in der Regel ersten postoperativen Tag entfernt werden, jedoch empfehlen wir die sofortige Neuanlage bei hämodynamischer Destabilisierung.

Während die invasive Blutdruckmessung bei kardiochirurgischen Patienten postoperativ angewendet werden soll, kann bei hämodynamisch stabilen Patienten laut Leitlinie auch eine NIBP angewendet werden [1]. Zu beachten ist hier aber die fehlende Möglichkeit, rasch und wiederholt arterielle Blutgasanalysen durchzuführen. Diese empfiehlt die Leitlinie innerhalb von 30 min nach Aufnahme und auch grundsätzlich bei beatmeten Patienten durchzuführen.

Eine relevante Limitation der NIBP stellen nicht pulsatile Flussverhältnisse bei Herzunterstützungssystemen wie der venoarteriellen extrakorporalen Membranoxygenierung (v-a-ECMO, ECLS) dar, die somit die invasive Blutdruckmessung notwendig machen.

\section{AUS DER PRAXIS}

\section{Harlekin-Syndrom}

Bei Anwendung perkutaner v-a-ECMO-Systeme kann in bis zu 10\% der Fälle eine Minderperfusion mit Hypoxie besonders der oberen rechten Körperhälfte beobachtet werden. Dieses sogenannte Harlekin-Syndrom entsteht bei retrograder arterieller Perfusion über eine einliegende arterielle ECMO-Kanüle, wenn sich zwischen retrogradem ECMO-Fluss und antegradem Auswurf des Herzens beim Zusammentreffen der beiden gegeneinander gerichteten Flüsse eine Wasserscheide im Bereich des Aortenbogens ausbildet.

Mit einer invasiven Blutdruckmessung in der rechten $\mathrm{A}$. radialis kann mithilfe der Blutgasanalysen einer Hypoxie durch ein Harlekin-Syndroms detektiert werden. Zudem kann über die Abnahme von kapillären Blutgasanalysen im Seitenvergleich und zwischen Ober- und Unterkörper ein pathologischer Blutfluss ermittelt werden.

\section{TAKE HOME MESSAGE}

- Invasive Blutdruckmessung bei hämodynamisch instabilen und/oder beatmeten Patienten.

- Bei Intensivpatienten bietet sich in der Praxis aufgrund von oft schwierigen Gefäßverhältnissen die primär ultraschallgestützte Etablierung einer arteriellen Blutdruckmessung an.

Blutgasanalyse: Auch über einen Pulmonalarterienkatheter (PAK) oder zentralen Venenkatheter (ZVK) können Blutgasanalysen gewonnen werden. Diese ermöglichen die Bestimmung der gemischt-venösen $\left(\mathrm{SvO}_{2}\right)$ bzw. zentralvenösen $\left(\mathrm{ScvO}_{2}\right)$ Sauerstoffsättigung. Die Leitlinie betont hier, dass nur bei stabilen Patienten die $\mathrm{ScvO}_{2}$ statt der $\mathrm{SvO}_{2}$ zur Beurteilung des Sauerstoffangebotes herangezogen werden sollte [1]. Zudem sollten diese Parameter im Hinblick auf eine inadäquate Sauerstoffversorgung nur in Zusammenschau mit der Laktatkonzentration evaluiert werden [7]. Die Autoren empfehlen, bei postoperativen Komplikationen bevorzugt die der $\mathrm{SvO}_{2}$ in Betracht zu ziehen, welche die Anlage eines PAK indiziert.

\section{AUS DER PRAXIS \\ Globale Gewebehypoxie erkennen}

Die Blutgasanalysen aus einem Arterienkatheter und einem ZVK können in Zusammenschau Hinweise auf eine globale Hypoxie geben. Die Abnahme der $\mathrm{ScvO}_{2}$ unter $<70 \%$ deutet auf eine erhöhte periphere Sauerstoffentnahme aus dem arteriellen Blut hin. Die Erhöhung des Laktatwertes > 2 mmol// kann mit einer Zunahme anaerober Stoffwechselvorgänge bei insuffizienter Mikroperfusion verbunden sein.

Besteht bei einem hämodynamisch instabilen Patienten eine Kombination aus erniedrigter $\mathrm{ScvO}_{2}$, einer Erhöhung des Laktatwertes und einer therapiebedürftigen Hypotonie, so muss dies klinisch als starker Hinweis auf eine Kreislaufinsuffizienz mit reduziertem Herzzeitvolumen gesehen werden.

Zentraler Venendruck: Der Registrierung des ZVD kommt ebenfalls eine hohe Relevanz zu; sie soll mittels kontinuierlicher Kurvendarstellung erfolgen. Während der Absolutwert des ZVD nicht als Parameter des Volumenstatus genutzt werden soll, liefert aber der zeitliche Verlauf relevante Informationen zur aktuellen Kreislaufsituation und der weiteren Prognose [8]. Typische postoperative Komplikationen sind oft mit plötzlichen Änderungen des ZVD vergesellschaftet. Bei einer Perikardtamponade oder einer rechtsventrikulären Dysfunktion kann ein akuter Anstieg des ZVD beobachtet werden. Diese Diagnosen müssen dann durch weitergehende Diagnostik, wie der Echokardiografie, weiter überprüft werden. 
Dieses Beispiel zeigt, dass eine engmaschige Dokumentation und klinische Evaluation des ZVD notwendig sind.

KEY-POINTS BASISMONITORING

- Postoperativ kontinuierliche EKG- (mindestens II und $V_{5}$ ) mit ST-Strecken-Überwachung.

- Arterielle Druckmessung und BGAs bei instabilen/ beatmeten Patienten.

- Bewertung von Laktat und $\mathrm{SvO}_{2} / \mathrm{ScvO}_{2}$ in Zusammenschau.

- ZVD als Verlaufsbeurteilung statt Bewertung des Absolutwertes.

\section{Erweitertes hämodynamisches Monitoring}

Der hohe Anspruch an das hämodynamische Management von kardiochirurgischen Intensivpatienten bedingt den Bedarf an erweiterten Monitoringverfahren, welche etwaige Therapieentscheide unterstützen können. Ein erweitertes hämodynamisches Monitoring soll dabei nur in Verbindung mit einem zielorientierten Behandlungskonzept zum Einsatz kommen [9].

\section{Merke \\ Erweitertes Monitoring nur in Verbindung mit ziel- orientierter Therapie!}

Echokardiografie: Besondere Anforderungen an den Anwender stellt hier sowohl die transthorakale als auch die transösophageale Echokardiografie. Die Echokardiografie wird in der aktuellen Leitlinie zur Diagnostik einer hämodynamischen Störung und zum Therapiemonitoring klar empfohlen und auch in der Praxis zunehmend eingesetzt [3]. Dies setzt eine entsprechende Anwenderkenntnis und die darauffolgende Dokumentation der Untersuchung voraus. Die klinischen Vorteile der direkten Visualisierung und Bestimmung sowohl der myokardialen als auch der Klappenfunktion $[10,11]$ rechtfertigen hier jedoch die aufwendige Ausbildung der ärztlichen Mitarbeiterinnen und Mitarbeiter einer kardiochirurgischen Intensivstation.

Die Autoren empfehlen die umfassende Schulung des ärztlichen Personals in den Techniken der Ultraschalldiagnostik und Umsetzung eines standardisierten Untersuchungsablaufs, um die Vergleichbarkeit der intermittierend erhobenen Ultraschallbefunde auch zwischen verschiedenen Untersuchern zu gewährleisten.

Pulmonalarterienkatheter: Der PAK stellt weiterhin ein nützliches und geschätztes hämodynamisches Monitoringverfahren dar [12], obgleich Echokardiografie und Thermodilutions-/Pulskonturanalysen eine immer größe- re Rolle im klinischen Alltag einnehmen. Der PAK ermöglicht die Erfassung diverser Funktionsparameter, von den intrakardialen Druckparametern hin bis zur Beurteilung des Herzzeitvolumens und der Bestimmung des $\mathrm{SvO}_{2}$.

Besonders bei rechtskardialer Dysfunktion oder pulmonalarteriellem Hypertonus kann der Pulmonalarterienkatheter relevante Informationen zur aktuellen kardialen Funktion liefern. So zeigt sich bei einer pulmonalen Hypertonie ein erhöhter systolischer rechtsventrikulärer (RV)-Druck, während eine rechtsventrikuläre diastolische Dysfunktion mit gestörter RV-Compliance durch einen erhöhten enddiastolischen RV-Druck zu erkennen ist. Da rechtsventrikuläre Dysfunktion und pulmonaler Hypertonus relevante Komorbiditäten bei vielen alten und chronisch herzinsuffizienten Patienten sind, kommt dem PAK bei postoperativen Komplikationen bei diesen Patienten eine hohe diagnostische und therapiesteuernde Relevanz zu.

\section{AUS DER PRAXIS}

\section{Anwendung des Pulmonalarterienkatheters}

Die risikobehaftete Anlage und die Interpretation der mithilfe eines Pulmonalarterienkatheters gewonnenen Befunde erfordern eine fundierte klinische Expertise und eine kritische Indikationsstellung. Bei Hochrisikopatienten mit vorbekannter rechtsventrikulärer Funktionseinschränkung, pulmonalarteriellen Hypertonie oder Low cardiac Output Syndrome (LCOS) wird der PAK zur gezielten Diagnostik und Steuerung der Intensivtherapie klar empfohlen.

Im Vergleich zu Alternativverfahren ist der PAK mit einer höheren Invasivität und relevanten Risiken assoziiert. Dennoch konnte bei Patienten mit moderatem bis hohem Risiko eine Reduktion von Morbidität und Letalität für eine PAK-basierte hämodynamische Therapie gezeigt werden $[13,14]$. Daher empfiehlt die Leitlinie den PAKEinsatz bei Hochrisikopatienten, besonders solchen mit Rechtsherzdysfunktion, pulmonalarteriellem Hypertonus oder linksventrikulärem Vorwärtsversagen mit inadäquatem Herzminutenvolumen und konsekutiver Gewebsminderperfusion [1].

Passive Leg Raise Test (PLR): Des Weiteren erwähnenswert ist die in der Leitlinie dokumentierte hohe klinische Relevanz des PLR zur Bestimmung der Volumenreagibilität. So soll bei herzchirurgischen Patienten hierfür der PLR eingesetzt werden. Im klinischen Alltag scheint der PLR besonders relevant, da er ohne irreversible Volumenlast schnell, bettseitig, nichtinvasiv und ohne großen apparativen Aufwand durchführbar ist und einen unmittelbaren Informationsgewinn bietet. 
\ Tab. 1 Zielparameter der hämodynamischen Therapie [1].

\begin{tabular}{|c|c|}
\hline Parameter & Grenzwert \\
\hline Sauerstoffsättigung & $\mathrm{ScvO}_{2} \geq 70 \%$ oder $\mathrm{SvO}_{2} \geq 65 \%^{*}$ \\
\hline MAD & $\geq 65 \mathrm{mmHg}$ \\
\hline SVI & $>35 \mathrm{ml} / \mathrm{m}^{2}$ \\
\hline SVV oder PPV & $<10-13 \%$ \\
\hline ZVD & $<15 \mathrm{mmHg}^{* *}$ \\
\hline LV-EDAI & $6-9 \mathrm{~cm}^{2} / \mathrm{m}^{2}$ \\
\hline RV-LV-Index & $<1$ \\
\hline GEDVI & $640-800 \mathrm{ml} / \mathrm{m}^{2 * *}$ \\
\hline PAOP & $15-18 \mathrm{mmHg}$ \\
\hline Diurese & $>0,5 \mathrm{ml} / \mathrm{kgKG} / \mathrm{h}$ \\
\hline Laktat & $\leq 2 \mathrm{mmol} / \mathrm{l}$ \\
\hline \multicolumn{2}{|c|}{$\begin{array}{l}\text { * Bei Werten der } \mathrm{ScvO}_{2} / \mathrm{SvO}_{2} \geq 80 \% \text { gibt es Hinweise, dass dies mit einer } \\
\text { verminderten Sauerstoffausschöpfung und mit einem schlechten Outcome } \\
\text { assoziiert sein könnte [17], vor allem, wenn gleichzeitig ein erhöhter Laktat- } \\
\text { wert } \geq 2 \mathrm{mmol} / \text { I vorliegt. }\end{array}$} \\
\hline \multicolumn{2}{|c|}{$\begin{array}{l}\text { ** Individuelle Grenzwerte können nach Volumenoptimierung durch TTE/TEE } \\
\text { oder dynamische Parameter bestimmt werden. }\end{array}$} \\
\hline \multicolumn{2}{|c|}{$\begin{array}{l}\text { Abkürzungen: GEDVI = globaler enddiastolischer Volumenindex; } \mathrm{LV} \text {-EDAI = } \\
\text { Left ventricular enddiastolic Area Index; } \mathrm{MAD}=\text { mittlerer arterieller Blutdruck; } \\
\text { PAOP = pulmonalarterieller Okklusionsdruck; PPV = Pulsdruckvarianz; RV-LV- } \\
\text { Index = rechtsventrikulärer zu linksventrikulärem Diameter-Index; } \mathrm{ScvO}_{2}= \\
\text { zentralvenöse Sauerstoffsättigung; } \mathrm{SVI} \text { = Schlagvolumenindex; } \mathrm{SVO}_{2}=\text { ge- } \\
\text { mischt-venöse Sauerstoffsättigung; SVV = Schlagvolumenvarianz; TEE = } \\
\text { transösophageale Echokardiografie; } \mathrm{TTE}=\text { transthorakale Echokardiografie; } \\
\text { ZVD = zentraler Venendruck }\end{array}$} \\
\hline
\end{tabular}

TIPP

Passive Leg Raise Test (PLR) - einfacher Test der Volumenreagibilität!

Transpulmonale Thermodilution und Pulskonturanalyse: Diese Verfahren ermöglichen ebenfalls die Erfassung einer Vielzahl von hämodynamischen Parametern, deren Nutzen im Zusammenhang mit einer zielorientierten Therapie zu sehen sind (für eine ausführliche Darstellung der Parameter und deren Interpretation verweisen die Autoren aufgrund des Umfangs auf einschlägige Fachliteratur). Im Vergleich zum PAK sind diese Verfahren mit einer geringeren Invasivität verbunden. Basierend auf verschiedenen Studien $[15,16]$ schätzt die Leitlinie die transpulmonale Thermodilution und die kalibrierte Pulskonturanalyse dem PAK gegenüber als äquivalentes Monitoringverfahren ein [1].

Dennoch bestehen relevante Limitationen des Verfahrens. Nach Kalibrierung der Pulskonturanalyse mittels transpulmonaler Thermodilution machen Veränderungen einer Katecholamin- oder Volumentherapien die erneute Kalibrierung notwendig. In der Praxis kann dies im Verlauf mit einer Vielzahl engmaschig durchzuführender
Kalibrierungen einhergehen. Somit kann die Pulskonturanalyse als Trendmonitor zwischen den einzelnen Kalibrationen betrachtet werden. Anzumerken ist zudem die grundsätzlich eingeschränkte Beurteilbarkeit der Pulskonturanalyse bei ausgeprägten Arrhythmien, Aortenklappeninsuffizienz und einer peripheren arteriellen Verschlusserkrankung (pAVK).

\section{KEY-POINTS ERWEITERTES MONITORING}

- Die Echokardiografie erfordert adäquate Personalschulung.

- PAK-Einsatz besonders bei Hochrisikopatienten.

- Die kalibrierte Pulskonturanalyse ist gegenüber dem PAK als äquivalentes Monitoringverfahren anzusehen.

- PLR liefert mit wenig Aufwand und Risiko Informationen über die Volumenreagibilität.

\section{Zielparameter der hämodynamischen Therapie}

Die Anwendung von Basis- und erweitertem hämodynamischem Monitoring ermöglicht die zielorientierte Kreislauftherapie. In diesem Zusammenhang definiert die aktuelle Leitlinie Zielparameter ( $\bullet$ Tab. 1), welche im Rahmen der Therapiemaßnahmen anzustreben sind [1]. In der klinischen Praxis gilt es jedoch, diese allgemeinen Zielparameter im klinischen Kontext zu den individuellen demografischen Besonderheiten und Komorbiditäten des Patienten betrachten.

\section{Volumentherapie}

Aufgrund des bei herzchirurgischen Patienten in der frühen postoperativen Phase häufig auftretenden relativen oder absoluten Volumenmangels gibt die Leitlinie Empfehlungen zur Volumentherapie und der Frage nach dem richtigen Volumenersatzmittel [1]. Hervorzuheben ist der Expertenkonsens, dass aufgrund der unzureichenden Evidenzlage keine Empfehlung zur Art des Volumenersatzes (kristalloid versus kolloidal) gegeben werden kann. Bei der Wahl der Therapeutika sind die behördlichen Zulassungsbeschränkungen für die Gruppe der Intensivpatienten besonders zu beachten. Dennoch kann laut Leitlinie der Ausgleich einer Hypovolämie und eine hämodynamische Stabilisierung mit künstlichen kolloidalen Volumenersatzmitteln vorgenommen werden [18-21].

Die therapeutische Anwendung von Albumin ist aufgrund der aktuellen Diskussionen um künstliche Kolloide zunehmend klinisch relevant. So zeigen zahlreiche Studien bei herzchirurgischen und allgemein kardiovaskulär erkrankten Patienten einen Zusammenhang zwischen perioperativer Hypalbuminämie und einer erhöhten postoperati- 
- Tab. 2 Dosierungsempfehlungen Vasoaktiva/Inotropika [1].

\begin{tabular}{|l|l|l|}
\hline Substanz & Initialbolus & Dosierungsraten \\
\hline Dobutamin & keiner & $2-20 \mu \mathrm{gg} / \mathrm{kgKG} / \mathrm{min}(\beta+)$ \\
\hline Dopamin & keiner & $3-5 \mu \mathrm{g} / \mathrm{kgKG} / \mathrm{min}(\beta+)$ \\
& & $>5 \mu \mathrm{g} / \mathrm{kgKG} / \mathrm{min}(\beta+/ \alpha+)$ \\
\hline Milrinon & $25-75 \mu \mathrm{g} / \mathrm{kgKG}$ über $10-20 \mathrm{~min}$ (optional) & $0,375-0,75 \mu \mathrm{m} / \mathrm{kgKG} / \mathrm{min}$ \\
\hline Enoximon & $0,5-1,0 \mathrm{mg} / \mathrm{kgKG}$ über $5-10 \mathrm{~min}$ (optional & $1-5 \mu \mathrm{mg} / \mathrm{kgKG} / \mathrm{min}$ \\
\hline Levosimendan & $12 \mu \mathrm{g} / \mathrm{kgKG} \mathrm{über}$ & $0,1 \mu \mathrm{g} / \mathrm{kgKG} / \mathrm{min}$ \\
\hline & $10 \mathrm{~min}$ (optional) & Reduktion bis $0,05 \mathrm{oder}$ \\
\hline Adrenalin & Reanimation: Bolus $1 \mathrm{mg}$ & Erhöhung bis $0,2 \mu \mathrm{mg} / \mathrm{kgKG} / \mathrm{min}$ \\
\hline Noradrenalin & Wiederholung nach 3-5 min & $0,05-0,5 \mu \mathrm{g} / \mathrm{kgKG} / \mathrm{min}$ \\
\hline Vasopressin & keiner & $0,2-1,0 \mu \mathrm{g} / \mathrm{kgKG} / \mathrm{min}$ \\
\hline Nitroglycerin & keiner & $0,5-1-(4)$ IE $/ \mathrm{h}$ \\
\hline Nitroprussidnatrium & Beginn mit $10-20 \mu \mathrm{g} / \mathrm{min}$, Steigerung bis $200 \mu \mathrm{g} / \mathrm{min}$ & \\
\hline
\end{tabular}

ven Komplikationsrate [22]. Mit dem Hinweis auf die Empfehlungen der Bundesärztekammer kann Albumin zur Therapie einer Hypovolämie und zur hämodynamischen Stabilisierung eingesetzt werden. Bei erwachsenen, nicht septischen Intensivpatienten sollen Albuminlösungen nicht angewandt werden, solange therapeutische Alternativen nicht ausgeschöpft sind.

\section{KEY-POINTS VOLUMENTHERAPIE}

- Keine abschließende Empfehlung zur Art des Volumenersatzes.

- Künstliche Kolloide und Albuminlösungen können zur hämodynamischen Stabilisierung bei Hypovolämie eingesetzt werden.

\section{Medikamentöse Kreislauftherapie}

Zur Erreichung der hämodynamischen Zielparameter stehen diverse Vasopressoren, Vasodilatatoren und Inotropika zur Verfügung. Die Leitlinie enthält neben der Diskussion der einzelnen Substanzen modifizierte Dosierungsempfehlungen, unter anderem basierend auf der ESCLeitlinie [31] für die akute Herzinsuffizienz ( Tab. 2; [1]).

Klinisch relevant erscheint hier, dass unter allen Inotropika bisher nur für den Kalziumsensitizer Levosimendan ein Überlebensvorteil, besonders bei präoperativer Anwendung vor Eintritt eines LCOS, gezeigt werden konnte [23, 24]. Besonders bei Therapiebeginn oder bei Gabe eines Bolus bedingt Levosimendan eine Vasodilatation mit z. T. klinisch relevantem Blutdruckabfall. Wurde bei Patienten mit einem arteriellen Blutdruck unter $100 \mathrm{mmHg}$ die Levosimendantherapie mittels Bolusgabe begonnen, zeigte sich eine erhöhte Letalität [25]. Somit sollte in der Praxis bei hypotensiven Patienten auf einen Initialbolus verzichtet werden.

Merke

Relevante Vasodilatation bei Therapie mit Levosimendan!

\section{Therapie der postoperativen Herzinsuffizienz}

Eine postoperative Linksherzinsuffizienz trägt wesentlich zu Mortalität und Morbidität bei. Die akute Linksherzinsuffizienz manifestiert sich mit einer plötzlichen Abnahme des Herzzeitvolumens und der konsekutiven Abnahme der systemischen Perfusion. Das klinische Bild wird bestimmt durch katecholaminpflichtige Hypotonie, Tachykardie und zunehmende Einschränkung der Oxygenierung. Dazu kommt ein pulmonaler Rückstau des Blutflusses mit begleitendem interstitiellem bis alveolärem Lungenödem.

Die perioperative Myokardschädigung, die Druck- bzw. Volumenüberlastung des linksventrikulären Myokards, aber auch das Myocardial Stunning (langsam rückläufige Kontraktilitätseinschränkung nach Reperfusion des Myokards) sind häufige Auslöser einer Linksherzinsuffizienz. Auch ein früher Verschluss eines linksventrikulären Bypasses kann eine akute Herzinsuffizienz bedingen.

Entsprechend der Empfehlung der Leitlinie soll bei Linksherzinsuffizienz vor weiteren medikamentösen oder maschinellen Maßnahmen eine Vorlastoptimierung erfolgen, an welche sich bei unzureichendem Effekt eine Va- 


\section{FALLBEISPIEL}

\section{Bypass-Frühverschluss}

Etwa 1 Stunde nach Aufnahme eines intubierten, sedierten und beatmeten Patienten nach komplikationsloser LIMA-LAD-ACB-Operation kommt es auf Intensivstation zu einer plötzlichen hämodynamischen Instabilität mit Tachykardie, Hypotonie und steigender Noradrenalinpflichtigkeit bis $0,45 \mu \mathrm{g} / \mathrm{kgKG} / \mathrm{min}$. Eine arterielle BGA zeigt eine progrediente Laktatazidose und Oxygenierungsstörung bei stabilen Hb-Werten. Trotz Eskalation einer positiv inotropen Therapie mit Dobutamin bis $8 \mu \mathrm{g} / \mathrm{kgKG} / \mathrm{min}$ kann die Noradrenalin-Dosis nicht reduziert werden, und der Patient zeigt eine zunehmende akrale Minderperfusion aller Extremitäten. Parallel wird bettseitig eine transösophageale Echokardiografie durchgeführt, welche eine neu aufgetretene Akinesie des interventrikulären Septums und der LV-Vorderwand mit hochgradiger Reduktion der linksventrikulären Ejektionsfraktion zeigt.

Bei Verdacht auf akute Linksherzinsuffizienz aufgrund eines Frühverschlusses des angelegten Bypasses erfolgt die operative Bypassrevision. sopressor- und/oder Inotropikatherapie anschließen sollte [1]. In der Praxis setzt dies eine genaue Kenntnis des Volumenstatus und der aktuellen Vorlast voraus, um eine ungezielte Volumentherapie, mit der Gefahr der Volumenüberladung, zu verhindern.

Als Vasopressoren sollten Noradrenalin und/oder Vasopressin eingesetzt werden. Bei Hypertonie kann keine klare Empfehlung für einen spezifischen Vasodilatator gegeben werden. Klinisch relevant ist auch die Empfehlung, zur Prävention hämodynamischer Komplikationen bei Patienten mit hochgradig eingeschränkter linksventrikulärer Funktion oder LCOS Levosimendan einzusetzen [2628]. Dies gilt es jedoch unter Beachtung aktueller Studienergebnisse [29] kritisch zu bewerten, da für die Levosimendantherapie bei postoperativer linksventrikulärer Dysfunktion keine Senkung der 30-Tage-Mortalität gezeigt werden konnte.

Die Therapie der Rechtsherzinsuffizienz stützt sich auf die folgenden drei Prinzipien:

- Gewährleistung eines adäquaten koronaren Perfusionsdruckes,

- Kontraktilitätssteigerung des rechten Ventrikels und

- Reduktion eines erhöhten pulmonalvaskulären Widerstandes (PVR).

Zusätzlich sollte eine angemessene Vorlast sichergestellt werden [30].

Die Volumentherapie und Vorlastoptimierung erfordern die engmaschige Therapiekontrolle mittels der diskutierten Maßnahmen wie ZVD, Pulmonalarterienkatheter, transthorakaler oder transösophagealer Echokardiografie. Ein ausreichender Perfusionsdruck sollte mittels Noradrenalin und/oder Vasopressingabe erzielt werden.

Bei schwerer pulmonalarterieller Hypertonie mit Rechtsherzdysfunktion kann als Alternative zu Noradrenalin auch Vasopressin eingesetzt werden, mit dem Ziel des verbesserten Verhältnisses zwischen pulmonalvaskulären und systemischen Gefäßwiderstandes. Die Leitlinie spricht für diesen Zusammenhang jedoch aufgrund einer niedrigen Evidenzlage nur eine schwache Empfehlung aus [1].

Ist ein ausreichender systemischer Perfusionsdruck sichergestellt, sollte die Therapie mit Nitraten und/oder inhalativen Vasodilatatoren erfolgen, um den pulmonalarteriellen Druck zu senken und so eine Entlastung des rechtsventrikulären Myokards zu bewirken. Besteht Bedarf für eine inotrope Therapie, können Dobutamin, Phosphodiesterase-III-Hemmer oder Levosimendan eingesetzt werden. Stellt sich die Rechtsherzinsuffizienz als therapierefraktär dar, kann nach Leitlinienempfehlung schließlich die Gabe von Adrenalin erwogen werden [31].

\section{Fazit}

Mit der neuesten Fassung der S3-Leitlinie zur intensivmedizinischen Versorgung herzchirurgischer Patienten - Hämodynamisches Monitoring und Herz-Kreislauf [1] steht ein umfassendes Werk zur Planung und Umsetzung von Therapiestrategien im klinischen Alltag zur Verfügung. Es finden sich nicht nur wissenschaftliche Informationen zu allen relevanten Teilbereichen der kardiochirurgischen Intensivmedizin, sondern zudem auch Handlungsempfehlungen bei häufigen Pathologien wie der Links-/und Rechtsherzinsuffizienz. Während dieser Artikel eine selektive Übersicht über klinisch relevante Aussagen der Leitlinie darstellt, kann eine direkte Auseinandersetzung mit der Leitlinie die eigene klinische Praxis sehr bereichern.

\section{KERNAUSSAGEN}

- Gemischt-venöse und/oder zentralvenöse Sättigung in Zusammenschau mit Laktatwert bewerten.

- Ein erweitertes Monitoring sollte mit einer zielorientierten Therapie verbunden sein.

- Künstliche Kolloide und Albumin spielen eine wichtige Rolle in der Volumenersatztherapie.

- Levosimendan kann eine schwere Hypotonie bedingen.

- Die Therapie der Rechtsherzinsuffizienz stützt sich auf adäquaten Perfusionsdruck, Kontraktilitätsverbesserung und Senkung eines erhöhten pulmonalvaskulären Widerstandes (PVR). 


\section{Interessenkonflikt}

D. S. gibt an, dass kein Interessenkonflikt besteht. F.D. hat Referentenhonorare im Rahmen des Forums für medizinische Fortbildung erhalten.

\section{Autorinnen/Autoren}

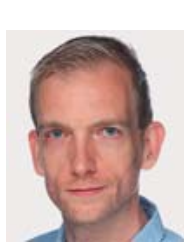

\section{David Sander}

Dr. med., Jahrgang 1981. 2003-2010 Studium der Humanmedizin an den Universitäten Budapest, Ulm, Wien und Hamburg. 2011-2016 Facharztweiterbildung, 2016-2019 Facharzt für Anästhesiologie. 2019-2020 Bereichsfacharzt Intensivmedizin. Seit 2020 Oberarzt der Klinik für Anästhesiologie und Operative Intensivmedizin, Uniklinik Köln.

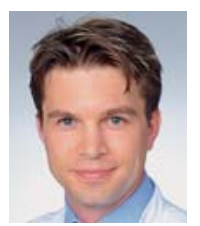

\section{Fabian Dusse}

Dr. med. MHBA, Jahrgang 1976. 2004-2012 Facharztausbildung und Funktionsoberarzt, Klinik für Anästhesiologie und Intensivmedizin, Universitätsklinikum Essen. 2012-2015 Oberarzt, Leiter der Intensivstationen, Klinik für Thorax- und Kardiovaskuläre Chirurgie, Universitätsklinikum Essen. 2015-2018 Oberarzt, Klinik für Anästhesiologie und operative Intensivmedizin, Krankenhaus KölnMerheim, Kliniken der Stadt Köln.

\section{Korrespondenzadresse}

\section{Dr. med. David Sander}

Klinik für Anästhesiologie und Operative Intensivmedizin Uniklinik Köln

Kerpener Straße 62

50937 Köln

Deutschland

david.sander@uk-koeln.de

\section{Literatur}

[1] Habicher M, Zajonz T, Bauer et al. S3-Leitlinie zur intensivmedizinischen Versorgung herzchirurgischer Patienten - Hämodynamisches Monitoring und Herz-Kreislauf. AWMF Register Nr. 001/016. Im Internet (Stand: 07.09.2020): https://www. awmf.org/leitlinien/detail/I/001-016.html

[2] Funcke S, Sander M, Goepfert MS et al. Practice of hemodynamic monitoring and management in German, Austrian, and Swiss intensive care units: the multicenter cross-sectional ICU-CardioMan Study. Ann Intensive Care 2016; 6: 49

[3] Kastrup M, Carl M, Spies C et al. Clinical impact of the publication of S3 guidelines for intensive care in cardiac surgery patients in Germany: results from a postal survey. Acta Anaesthesiol Scand 2013; 57: 206-213

[4] London MJ, Hollenberg M, Wong MG et al. Intraoperative myocardial ischemia: localization by continuous 12 -lead electrocardiography. Anesthesiology 1988; 69: 232-241

[5] Bur A, Hirschl MM, Herkner $\mathrm{H}$ et al. Accuracy of oscillometric blood pressure measurement according to the relation between cuff size and upper-arm circumference in critically ill patients. Crit Care Med 2000; 28: 371-376
[6] Hollenberg SM, Ahrens TS, Annane D et al. Practice parameters for hemodynamic support of sepsis in adult patients: 2004 update. Crit Care Med 2004; 32: 1928-1948

[7] Hu BY, Laine GA, Wang $S$ et al. Combined central venous oxygen saturation and lactate as markers of occult hypoperfusion and outcome following cardiac surgery. J Cardiothorac Vasc Anesth 2012; 26: 52-57

[8] Williams JB, Peterson ED, Wojdyla D et al. Central venous pressure after coronary artery bypass surgery: does it predict postoperative mortality or renal failure? J Crit Care 2014; 29: 1006-1010

[9] Aya HD, Cecconi M, Hamilton M et al. Goal-directed therapy in cardiac surgery: a systematic review and meta-analysis. $\mathrm{Br}$ J Anaesth 2013; 110: 510-517

[10] Hainer C, Bernhard M, Scheuren K et al. [Echocardiography during acute hemodynamic instability]. Anaesthesist 2006; 55: 1117-1131

[11] Treskatsch S, Habicher M, Sander M. Echokardiografie als Monitoring auf der Intensivstation? Anaesthesiol Intensivmed Notfallmed Schmerzther 2014; 49: 708-717

[12] Trottier S], Taylor RW. Physicians' attitudes toward and knowledge of the pulmonary artery catheter: Society of Critical Care Medicine membership survey. New Horiz 1997; 5: 201-206

[13] Gurgel ST, do Nascimento P jr. Maintaining tissue perfusion in high-risk surgical patients: a systematic review of randomized clinical trials. Anesth Analg 2011; 112: 1384-1391

[14] Hamilton MA, Cecconi M, Rhodes A. A systematic review and meta-analysis on the use of preemptive hemodynamic intervention to improve postoperative outcomes in moderate and high-risk surgical patients. Anesth Analg 2011; 112: $1392-$ 1402

[15] Gödje O, Höke K, Goetz AE et al. Reliability of a new algorithm for continuous cardiac output determination by pulse-contour analysis during hemodynamic instability. Crit Care Med 2002; 30: $52-58$

[16] Zöllner C, Haller M, Weis M et al. Beat-to-beat measurement of cardiac output by intravascular pulse contour analysis: a prospective criterion standard study in patients after cardiac surgery. J Cardiothorac Vasc Anesth 2000; 14: 125-129

[17] Laine GA, Hu BY, Wang $S$ et al. Isolated high lactate or low central venous oxygen saturation after cardiac surgery and association with outcome. J Cardiothorac Vasc Anesth 2013; 27: 1271-1276

[18] Annane D, Siami S, Jaber S et al. Effects of fluid resuscitation with colloids vs. crystalloids on mortality in critically ill patients presenting with hypovolemic shock: the CRISTAL randomized trial. JAMA 2013; 310: 1809-1817

[19] Gillies MA, Habicher M, Jhanji S et al. Incidence of postoperative death and acute kidney injury associated with i.v. $6 \%$ hydroxyethyl starch use: systematic review and meta-analysis. $\mathrm{Br}$ J Anaesth 2014; 112: 25-34

[20] Kim J-Y, Joung K-W, Kim K-M et al. Relationship between a perioperative intravenous fluid administration strategy and acute kidney injury following off-pump coronary artery bypass surgery: an observational study. Crit Care 2015; 19: 350

[21] Saw MM, Chandler B, Ho KM. Benefits and risks of using gelatin solution as a plasma expander for perioperative and critically ill patients: a meta-analysis. Anaesth Intensive Care 2012; 40: 17-32

[22] Karas PL, Goh SL, Dhital K. Is low serum albumin associated with postoperative complications in patients undergoing cardiac surgery? Interact Cardiovasc Thorac Surg 2015; 21: 777 786 
[23] Chen $\mathrm{Q}-\mathrm{H}$, Zheng R-O, Lin $\mathrm{H}$ et al. Effect of levosimendan on prognosis in adult patients undergoing cardiac surgery: a meta-analysis of randomized controlled trials. Crit Care 2017; 21: 253

[24] Sanfilippo F, Knight JB, Scolletta S et al. Levosimendan for patients with severely reduced left ventricular systolic function and/or low cardiac output syndrome undergoing cardiac surgery: a systematic review and meta-analysis. Crit Care 2017; 21: 252

[25] Packer M, Colucci W, Fisher L et al. Effect of levosimendan on the short-term clinical course of patients with acutely decompensated heart failure. JACC Heart Fail 2013; 1: 103-111

[26] Maharaj R, Metaxa V. Levosimendan and mortality after coronary revascularisation: a meta-analysis of randomised controlled trials. Crit Care 2011; 15: R140

[27] Harrison RW, Hasselblad V, Mehta RH et al. Effect of levosimendan on survival and adverse events after cardiac surgery: a meta-analysis. J Cardiothorac Vasc Anesth 2013; 27: 12241232

[28] Belletti A, Castro ML, Silvetti S et al. The Effect of inotropes and vasopressors on mortality: a meta-analysis of randomized clinical trials. Br J Anaesth 2015; 115: 656-675
[29] Landoni G, Lomivorotov VV, Alvaro G et al. Levosimendan for Hemodynamic Support after Cardiac Surgery. N Engl J Med 2017; 376: 2021-2031

[30] Winterhalter M, Antoniou T, Loukanov T. Management of adult patients with perioperative pulmonary hypertension: technical aspects and therapeutic options. Cardiology 2010; 116: 3-9

[31] Ponikowski P, Voors AA, Anker SD et al. 2016 ESC Guidelines for the diagnosis and treatment of acute and chronic heart failure: The Task Force for the diagnosis and treatment of acute and chronic heart failure of the European Society of Cardiology (ESC) developed with the special contribution of the Heart Failure Association (HFA) of the ESC. Eur Heart J 2016; 37: $2129-2200$

\section{Bibliografie}

Anästhesiol Intensivmed Notfallmed Schmerzther 2020; 55: 635-642

DOI 10.1055/a-1011-2078

ISSN $\quad 0939-2661$

(C) 2020. Thieme. All rights reserved.

Georg Thieme Verlag KG, Rüdigerstraße 14,

70469 Stuttgart, Germany 